\title{
Monitoring human papillomavirus prevalence in urine samples: a review
}

This article was published in the following Dove Press journal:

Clinical Epidemiology

II March 2013

Number of times this article has been viewed

\author{
Espen Enerly \\ Cecilia Olofsson \\ Mari Nygård \\ Department of Research, Cancer \\ Registry of Norway, Oslo, Norway
}

Correspondence: Espen Enerly Cancer Registry of Norway, PO Box 5313 Majorstuen 0304, Oslo, Norway Tel +472333 3956

Fax +472333 3956

Email espen.enerly@kreftregisteret.no
Abstract: Human papillomavirus (HPV) is the main cause of cervical cancer, and many countries now offer vaccination against HPV to girls by way of government-funded national immunization programs. Monitoring HPV prevalence in adolescents could offer a near-term biological measure of vaccine impact, and urine sampling may be an attractive large-scale method that could be used for this purpose. Our objective was to provide an overview of the literature on HPV DNA detection in urine samples, with an emphasis on adolescents. We searched the PubMed database using the terms "HPV" and "urine" and identified 21 female and 14 male study populations in which HPV prevalence in urine samples was reported, four of which included only asymptomatic female adolescents. We provide herein an overview of the recruitment setting, age, urine sampling procedure, lesion type, HPV assay, and HPV prevalence in urine samples and other urogenital samples for the studies included in this review. In female study populations, concordance for any HPV type and type-specific concordance in paired urine and cervical samples are provided in addition to sensitivity and specificity. We concluded that few studies on HPV prevalence in urine samples have been performed in asymptomatic female adolescent populations but that urine samples may be a useful alternative to cervical samples to monitor changes in HPV prevalence in females in the post-HPV vaccination era. However, care should be taken when extrapolating HPV findings from urine samples to the cervix. In males, urine samples do not seem to be optimal for monitoring HPV prevalence due to a low human genomic DNA content and HPV DNA detection rate compared to other urogenital sites. In each situation the costs and benefits of HPV DNA detection in urine compared to alternative monitoring options should be carefully considered.

Keywords: cervical cancer, HPV, surveillance, vaccination, virology, cervix

Human papillomavirus (HPV) is the main cause of cervical cancer. ${ }^{1,2}$ More than $35 \mathrm{HPV}$ types have been identified in the genital tract, and HPV16 and 18 are responsible for approximately $70 \%$ of cervical cancer. ${ }^{3,4}$ Two HPV vaccines, a quadrivalent vaccine that protects against HPV6, 11, 16, and 18 and a bivalent vaccine that protects against HPV16 and 18, have been developed and approved by the United States Food and Drug Administration (FDA) for females 9-25 years and 9-26 years of age, respectively.,6 The quadrivalent vaccine has also been approved by the FDA for males 9-26 years of age for the prevention of genital warts (caused by HPV6 and 11) and in both males and females 9-26 years of age for the prevention of precancerous anal lesions and anal cancer associated with the vaccine HPV types. ${ }^{7}$ However, in many countries the debate continues as to whether or not to offer the vaccine to boys in the framework of government-funded national immunization programs as is done for girls. ${ }^{8}$ Although the 
safety, immunogenicity, and efficacy of the two vaccines have been monitored closely in clinical trials, ${ }^{9-14}$ it is also necessary to monitor their impact in the general population. ${ }^{15}$ In addition to vaccine coverage and the incidence of cervical cancer and other HPV-related lesions, it is also recommended to monitor changes in HPV prevalence in the general population, particularly in females aged 15-20 years soon after initiation of sexual activity as this could offer a near-term biological measure of vaccine impact. ${ }^{16}$

HPV DNA detection in urine samples could be a viable alternative to detection in cervical samples for monitoring the impact of vaccination on overall and type-specific HPV prevalence. ${ }^{17,18}$ HPV DNA detection in urine samples is particularly attractive as a large-scale method among female adolescents as pelvic examination might not be feasible or ethically acceptable in this age group. HPV detection has also been performed in urine samples from males as well as at many urogenital sites. ${ }^{19}$ The urogenital sites most commonly sampled have been penile skin, specifically coronal sulcus and glans penis, and the urethra. ${ }^{20}$ In a recent review by Vorsters et al, ${ }^{17} 41$ published studies on HPV DNA in urine samples were evaluated with respect to factors that have an impact on HPV detection, such as urine sampling procedure, sample preparation, DNA extraction, and DNA amplification. They concluded that HPV DNA detection in urine is feasible and may become a useful tool but necessitates further improvement and standardization. In the same vein, Sehgal et $\mathrm{al}^{21}$ reviewed the pros and cons of using HPV DNA detection in urine samples for cervical cancer screening. ${ }^{21}$ One of their conclusions was that further research is needed to standardize and optimize the corresponding technology before recommending it as a mass screening tool for cervical cancer.

The aim of this literature review was to evaluate whether HPV DNA detection in urine samples would be a feasible approach for monitoring HPV prevalence in male and female adolescents in the general population. To this end, a list of relevant studies and their main characteristics, including HPV positivity in paired urine and cervical samples and their concordance is provided. Since only a few studies monitored HPV prevalence in urine samples from adolescents, we included relevant studies from older age groups as well. For details on factors that affect HPV DNA detection, such as urine sampling procedure, storage, centrifugation, DNA extraction, and assays for detection of human genomic and HPV DNA and an overview of the use of urine samples in a screening setting, we refer the reader to the reviews by Vorsters et $\mathrm{al}^{17}$ and Sehgal et al. ${ }^{21}$

\section{Materials and methods}

We used the terms "HPV" and "urine" to search the PubMed database in August 2012 for relevant literature. The identified studies were then individually evaluated to ascertain whether results on HPV prevalence in adolescents were reported. Studies in HIV-positive populations and renal allograft recipients were excluded as these patient groups have a reported increased risk of HPV infection. Studies that included paired urine and cervical samples were sought as they would allow for comparison of the presence of HPV in both samples. We therefore excluded female studies in which HPV DNA was detected in urine samples only, with the exception of the four studies including adolescents as they shed some light on the expected urinary HPV prevalence rates in this group. As few studies in asymptomatic male populations were found, we included the male populations of four studies despite the fact that they may be at greater risk of HPV infection: two studies on male partners of HPV-positive women, one on male partners of women with cervical cancer, and one on males with urethritis.

Whenever possible we extracted information on country, recruitment setting, age (range and mean), urine sampling procedure, total sample size, percentage of samples containing human genomic DNA, HPV assay used, proportion and type of cervical lesions, and availability of cervical sample. As a main result we report HPV prevalence in urine samples and cervical samples in female study populations. We also report sensitivity, ie, the probability of an HPV-positive urine sample given an HPV-positive cervical sample, and specificity, ie, the probability an HPV-negative urine sample given an HPV-negative cervical sample. Concordance for any HPV type and for HPV16 (and/or HPV18) was measured as the percentage of paired urine and cervical samples that yielded the same HPV result, ie, either both positive or both negative. In contrast, type-specific concordance (combined concordance for a group of HPV types) was measured only in the set of paired urine and cervical samples that were both positive for any HPV type. Within this smaller set of samples, type-specific concordance was calculated as the percent of paired urine and cervical samples that were positive for the same HPV type.

\section{Results}

\section{HPV prevalence in females}

Results from 21 female study populations from 17 publications that reported HPV prevalence in urine samples are summarized in Table 1. Four of these studies reported age-specific HPV prevalence in asymptomatic adolescents. 
Prusty et $\mathrm{al}^{22}$ and Manhart et $\mathrm{al}^{23}$ included females $18-25$ years of age, while only two studies, one from Scotland by O'Leary et $\mathrm{al}^{24}$ and one from India by Hussain et $\mathrm{al},{ }^{25}$ included females less than 18 years of age (Figure 1 and Table 1). The HPV prevalence (any type) varied from $1.1 \%$ in the age group $11-14$ years in the O'Leary et $\mathrm{al}^{24}$ study to $29.6 \%$ in the age group 20-21 years in the Manhart et $\mathrm{al}^{23}$ study. O'Leary et $\mathrm{al}^{24}$ reported the lowest percentage of samples $(83.6 \%)$ containing amplifiable human genomic DNA; Prusty et $\mathrm{al}^{22}$ reported the highest percentage (100\%).

We identified 14 studies that measured HPV positivity in paired urine and cervical samples from symptomatic females; ${ }^{18,22,26-37}$ of these studies, nine reported the age range and nine the mean age of the study population. These studies included women with symptoms that led to various gynecological complaints and women diagnosed with invasive cervical cancer. Urine and cervical samples were collected at gynecological, colposcopy, genitourinary, sexually transmitted disease, and adolescent clinics. In general, HPV prevalence increased with the severity of cytological or pathological findings, and this was observed both in urine samples and cervical samples. All studies that reported agespecific HPV prevalence showed lower prevalence in urine samples than paired cervical samples, with the majority of the studies reporting a 10\%-20\% lower HPV prevalence, save the studies by Rymark et $\mathrm{al}^{33}$ and Cuschieri et al ${ }^{18}$ (Figure 2). With the exception of studies by Rymark et $\mathrm{al}^{33}$ and Jacobson et al, ${ }^{31}$ which covered narrow age ranges (16-21 years and 11-20 years, respectively), the studies in this review generally reported HPV prevalence in a broad age range. Populations presenting cytological or histological pathology generally had a higher HPV prevalence compared to those with normal cytology (Figures 1 and 2).

\section{HPV concordance in paired urine and cervical samples}

The studies showed a $75 \%-100 \%$ concordance for any HPV type in paired urine and cervical samples (or $41 \%-93 \%$ by kappa agreement). Type-specific concordance was reported in five studies, showing $100 \%, 90.5 \%, 71.0 \%, 70.8 \%$, and $40.0 \%$ concordance. The latter estimates were calculated by including HPV-negative samples from either (not both) site, thereby reducing the type-specific concordance. Eight of the eleven studies published after 2000 also reported either type-specific concordance for HPV16 or HPV18, or concordance for the two types in combination. HPV16specific concordance in the studies reviewed varied from 64\%-100\%.
HPV16-specific concordance or concordance for any HPV type by severity of cervical lesion was reported in three studies. Daponte et $\mathrm{al}^{28}$ showed an increase in HPV16/18specific concordance with increasing severity. In contrast Alameda et $\mathrm{al}^{26}$ reported a higher concordance for any HPV type in low grade squamous intraepithelial lesion (LSIL) than in high grade squamous intraepithelial lesion (HSIL). Forslund et $\mathrm{al}^{29}$ showed neither an increase nor a decrease in concordance for any HPV type with increasing severity.

The only study that estimated sensitivity and specificity for HPV DNA detection in urine samples compared to cervical samples in a presumably asymptomatic, drop-in, sexual health service clinic population was published by Cuschieri et al ${ }^{18}$ who reported a sensitivity of $90.6 \%(95 \%$ confidence interval [CI] 79.3\%-96.9\%) and a specificity of $67.6 \%$ (95\% CI 50.2\%-82.0\%). In the studies with symptomatic populations, the sensitivity varied from $52.9 \%$ to $100 \%$. Specificity varied from $66.7 \%$ to $100 \%$.

\section{HPV positivity in males}

We identified 14 male study populations from 13 publications that reported HPV positivity in urine samples and at least one other urogenital site (Table 2). Only the studies by Lazcano-Ponce et $\mathrm{al}^{38}$ and Cuschieri et al ${ }^{18}$ included males less than 18-years old. The 117 males aged 16-25 years in the Cuschieri et $\mathrm{al}^{18}$ study showed a $36.7 \%$ HPV prevalence in urine samples versus $29.1 \%$ in samples taken from the shaft of the penis. The study by Lazcano-Ponce et $\mathrm{al}^{38}$ included 120 healthy males aged 14-55 years in Mexico (43 males in the range $14-20$ years) and reported a $6.9 \%$ and $42.7 \% \mathrm{HPV}$ prevalence in urine samples and urethra samples, respectively. Similarly, HPV prevalence in the other studies listed in Table 2 was generally lower in urine samples than in other urogenital samples. For example, Weaver et $\mathrm{al}^{39}$ recruited 317 sexually active university students, aged 18-25 years, and measured HPV prevalence in urine samples as well as in samples from the glans, penile shaft, scrotum, and foreskin. Samples taken from the foreskin showed the highest prevalence $(28.1 \%)$, while urine samples showed the lowest prevalence $(5.8 \%)$.

The percentage of urine samples in which human genomic DNA could be detected varied substantially between the studies. While Weaver et $\mathrm{al}^{39}$ detected human genomic DNA in $99.7 \%$ of urine samples and $94.0 \%-98.3 \%$ of samples from other sites, Giuliano et al only detected human genomic DNA in 51.3\% of urine samples and $84.0 \%-98.0 \%$ of samples from other sites, with semen samples having the highest human genomic DNA detection rate. In the study by Hernandez et al, ${ }^{40}$ semen 
Table I Studies with human papillomavirus DNA detected in urine samples from asymptomatic adolescents (A) and in paired urine and cervical samples from symptomatic females all ages (B)

\begin{tabular}{|c|c|c|c|c|c|c|c|}
\hline Author & Country & \multicolumn{2}{|c|}{$\begin{array}{l}\text { Recruitment setting/ } \\
\text { patient characteristics }\end{array}$} & $\begin{array}{l}\text { Age, years } \\
\text { range (mean) }\end{array}$ & $\begin{array}{l}\text { Urine sampling } \\
\text { procedure }\end{array}$ & $\begin{array}{l}\text { HPV types - assays } \\
\text { (types detected) }^{\mathrm{a}}\end{array}$ & $\begin{array}{l}\text { Total } \\
\text { sample size }\end{array}$ \\
\hline \multicolumn{8}{|l|}{ Part A } \\
\hline $\begin{array}{l}\text { Prusty } \\
\text { et al }{ }^{22}\end{array}$ & India & \multicolumn{2}{|c|}{ Sexually naive college girls } & $18-25$ & & $\begin{array}{l}\text { In house } \mathrm{LI} \text { consensus } \\
\text { primers (any type }=- \text { ) }\end{array}$ & 100 \\
\hline $\begin{array}{l}\text { Manhart } \\
\text { et } \mathrm{al}^{23}\end{array}$ & USA & \multicolumn{2}{|c|}{$\begin{array}{l}\text { Randomly selected females from } \\
\text { the National Longitudinal Study } \\
\text { of Adolescent Health (Wave III) }\end{array}$} & $18-25(2 \mid .7)$ & Sampled at home & $\begin{array}{l}\text { PCR with primers and dot } \\
\text { blot with generic primers } \\
\text { (any type }=\geq 36 \text { types) }\end{array}$ & 3741 \\
\hline $\begin{array}{l}\text { O'Leary } \\
\text { et } \mathrm{al}^{24}\end{array}$ & Scotland & $\begin{array}{l}\text { Recruited } f \\
\text { and publicl) } \\
\text { Additional } \\
\text { early schoo } \\
\text { oversampli } \\
\text { age groups }\end{array}$ & $\begin{array}{l}\text { fm private } \\
\text { funded schools. } \\
\text { ecruitment among } \\
\text { leavers and } \\
\text { in older }\end{array}$ & $15-18$ & Sampled at clinic & $\begin{array}{l}\text { HPV INNO-LiPA } \\
\text { (any type }=27 \text { types, } \\
\text { HR-HPV }=18 \text { types) }\end{array}$ & $|34|$ \\
\hline $\begin{array}{l}\text { Hussain } \\
\text { et } \mathrm{al}^{25}\end{array}$ & India & \multicolumn{2}{|c|}{ Public school students } & $\begin{array}{l}8-17 \\
(14.1)\end{array}$ & Sampled in private & $\begin{array}{l}\text { PCR with MY primers } \\
\text { (any type } \geq 36 \text { types) }\end{array}$ & \\
\hline \multicolumn{8}{|l|}{ Part B } \\
\hline \multirow[t]{2}{*}{$\begin{array}{l}\text { Rymark } \\
\text { et } \mathrm{al}^{33}\end{array}$} & Sweden & $\begin{array}{l}\text { STD and } \\
\text { adolescent } \\
\text { clinic } \\
\text { patients }\end{array}$ & $\begin{array}{l}\text { Present or history } \\
\text { of genital warts }\end{array}$ & $|6-2|(\mid 8.6)$ & $\begin{array}{l}\text { After Pap with } \\
\text { a cotton swab, } \\
\text { urethral samples }\end{array}$ & $\begin{array}{l}\text { In-house PCR } \\
\text { (any type = five types) } \\
\text { Type-specific concordance } \\
\text { based on five types }\end{array}$ & 24 \\
\hline & & & $\begin{array}{l}\text { Gynecological } \\
\text { complains }\end{array}$ & $|5-2|(\mid 8.3)$ & & & 25 \\
\hline $\begin{array}{l}\text { Forslund } \\
\text { et } \mathrm{al}^{29}\end{array}$ & Sweden & \multicolumn{2}{|c|}{$\begin{array}{l}\text { Colposcopy clinic referral. } \\
\text { Suspected cytological changes }\end{array}$} & 17-79 (37) & $\begin{array}{l}\text { After gynecological } \\
\text { exam without } \\
\text { prior washing }\end{array}$ & $\begin{array}{l}\text { In-house PCR } \\
\text { (any type }=- \text { ) and } \\
\text { dot blot analysis. } \\
\text { Type-specific concordance } \\
\text { based on six types }\end{array}$ & $512^{g}$ \\
\hline $\begin{array}{l}\text { Strauss } \\
\text { et al }{ }^{37}\end{array}$ & UK & \multicolumn{2}{|c|}{$\begin{array}{l}\text { Randomly selected } \\
\text { genitourinary clinic patients }\end{array}$} & $16-57(26)$ & $\begin{array}{l}\text { Mid-stream } \\
\text { urine }\end{array}$ & $\begin{array}{l}\text { PCR with MY and } \\
\text { GP primers } \\
\text { (any type } \geq 36 \text { types) }\end{array}$ & 144 \\
\hline $\begin{array}{l}\text { Jacobson } \\
\text { et } \mathrm{al}^{31}\end{array}$ & USA & \multicolumn{2}{|c|}{$\begin{array}{l}\text { Consecutively enrolled STD } \\
\text { and adolescent clinic patients }\end{array}$} & $\mathrm{II}-20(17.5)^{\mathrm{h}}$ & First-void & $\begin{array}{l}\text { PCR with MY primers } \\
\text { (any type }=34 \text { types) and } \\
\text { hybrid capture probe B } \\
\text { Type-specific concordance } \\
\text { based on six HR types }\end{array}$ & 80 \\
\hline $\begin{array}{l}\text { Sellors } \\
\text { et } \mathrm{al}^{34}\end{array}$ & Canada & \multicolumn{2}{|c|}{$\begin{array}{l}\text { Colposcopy clinic referral. } \\
\text { Abnormal cytology }\end{array}$} & $(31.5)$ & $\begin{array}{l}\text { First-void urine after } \\
\text { self-sampled vulvar } \\
\text { and vaginal sample, } \\
\text { but prior to cervical } \\
\text { sampling }\end{array}$ & $\begin{array}{l}\text { HC II } \\
\text { (any type = } 17 \text { types) }\end{array}$ & 245 \\
\hline $\begin{array}{l}\text { Stanczuk } \\
\text { et al }{ }^{36}\end{array}$ & Zimbabwe & \multicolumn{2}{|c|}{$\begin{array}{l}\text { Invasive cancer patients } \\
\text { at gynecological clinic }\end{array}$} & $24-70(44)^{\mathrm{h}}$ & $\begin{array}{l}\text { Prior to cervical } \\
\text { sampling }\end{array}$ & $\begin{array}{l}\text { In-house PCR } \\
\text { (any type }=- \text { ) }\end{array}$ & 43 \\
\hline $\begin{array}{l}\text { Prusty } \\
\text { et } \mathrm{al}^{22}\end{array}$ & India & \multicolumn{2}{|c|}{$\begin{array}{l}\text { Gynecological out-patient } \\
\text { and family planning clinic } \\
\text { patients. Married and sexually } \\
\text { active. Complaints other than } \\
\text { gynecological }\end{array}$} & $18-35$ & $\begin{array}{l}\text { Prior to cervical } \\
\text { sampling using dry } \\
\text { "paper smear" }\end{array}$ & $\begin{array}{l}\text { In house } \mathrm{LI} \text { consensus } \\
\text { primers (any type }=- \text { ) }\end{array}$ & 55 \\
\hline
\end{tabular}




\begin{tabular}{|c|c|c|c|c|c|c|}
\hline $\begin{array}{l}\text { Samples with human } \\
\text { genomic DNA (\%) }\end{array}$ & $\begin{array}{l}\text { Lesions/HPV } \\
\text { types (\% lesions) }\end{array}$ & $\begin{array}{l}\text { HPV positivity } \\
\text { in urine samples (\%) }\end{array}$ & $\begin{array}{l}\text { HPV positivity in } \\
\text { cervical samples (\%) }\end{array}$ & $\begin{array}{l}\text { Concordance } \\
\text { (\%) }\end{array}$ & $\begin{array}{l}\text { Sensitivity } \\
\text { (\%) }\end{array}$ & $\begin{array}{l}\text { Specificity } \\
(\%)\end{array}$ \\
\hline \multirow[t]{2}{*}{$100(100)$} & All/any type & 6.0 & & & & \\
\hline & All/HPVI6 & 4.0 & & & & \\
\hline \multirow[t]{2}{*}{$3262(95.8)^{b}$} & All/any type & $26.9^{c}$ & & & & \\
\hline & All/HPVI6 & $5.8^{c, d}$ & & & & \\
\hline \multirow[t]{6}{*}{ II $2 \mid$ (83.6) } & All/any type & $1 . I^{c}$ & & & & \\
\hline & All/HR-HPV & $0.9^{\mathrm{c}, \mathrm{d}}$ & & & & \\
\hline & All/HPVI6/I8 & $0.0^{c, d}$ & & & & \\
\hline & All/any type & $15.2^{c}$ & & & & \\
\hline & All/HR-HPV & $12.6^{\mathrm{c,d}}$ & & & & \\
\hline & All/HPVI6/I8 & $6.5^{c, d}$ & & & & \\
\hline \multirow[t]{3}{*}{458} & All/any type & 3.3 & & & & \\
\hline & All/HPVI6 & 2.2 & & & & \\
\hline & All/HPVI8 & 0.4 & & & & \\
\hline \multirow[t]{5}{*}{$24(100)$} & All/any type & $70.8^{f}$ & 62.5 & 83.3 & 93.3 & 66.7 \\
\hline & All/Type-specific HPV & & & 100.0 & & \\
\hline & Normal (54) & & & & & \\
\hline & CINI (42) & & & & & \\
\hline & CIN2 (4) & & & & & \\
\hline \multirow[t]{5}{*}{$25(100)$} & All/any type & $16.0^{f}$ & 12.0 & 96.0 & 100 & 95.5 \\
\hline & Normal (80) & & & & & \\
\hline & CINI (I2) & & & & & \\
\hline & CIN2 (4) & & & & & \\
\hline & NA (4) & & & & & \\
\hline \multirow[t]{6}{*}{489 (95.5) } & All/any type & 38.2 & 49.3 & 77.1 & 65.6 & 88.3 \\
\hline & All/type-specific HPV & & & 90.5 & & \\
\hline & Benign/any type (64) & 24.8 & 32.1 & 78.7 & 55.4 & 89.7 \\
\hline & CIN I/2/3/any type (30) & 61.2 & 81.0 & 73.5 & 71.4 & 82.1 \\
\hline & Cancer/any type (I) & 50.0 & 75.0 & 75.0 & 66.7 & 100.0 \\
\hline & Genital warts/any type (5) & 73.9 & 78.3 & 78.3 & 83.3 & 60.0 \\
\hline $136(94.4)$ & All/any type & 65.4 & 77.9 & 75.7 & 76.4 & 73.3 \\
\hline \multirow[t]{7}{*}{$80(100)$} & All/any type & 75.0 & 90.0 & 82.5 & 81.9 & 87.5 \\
\hline & All/type-specific HPV & & & $40.0^{\circ}$ & & \\
\hline & All/HPVI6 & 11.2 & 21.3 & 82.5 & 35.3 & 95.2 \\
\hline & All/HPVI8 & 23.3 & 9.6 & 98.8 & 100 & 98.6 \\
\hline & Normal/any type (62) & 65.3 & 83.7 & & & \\
\hline & ASCUS/any type (19) & 86.7 & 100 & & & \\
\hline & LSIL-HSIL/any type (19) & 100 & 100 & & & \\
\hline \multirow[t]{5}{*}{$200(81.6)$} & All/any type & 34.5 & 62.5 & $41.0^{k}$ & & \\
\hline & LSIL (27) & & & & & \\
\hline & HSIL (36) & & & & & \\
\hline & ASCUS (37) & & & & & \\
\hline & Cancer (I) & & & & & \\
\hline \multirow[t]{4}{*}{$35(8 \mathrm{I} .4)$} & All/any type & 88.6 & 100 & 88.6 & 88.6 & \\
\hline & All/type-specific HPV & & & 71.0 & & 83.3 \\
\hline & All/HPVI6 & 54.3 & 65.7 & 77.1 & 73.9 & 93.3 \\
\hline & All/HPVI8 & 11.4 & 14.3 & 85.7 & 40.0 & \\
\hline \multirow[t]{2}{*}{$55(100)$} & All/any type & 9.1 & 9.1 & 100 & & \\
\hline & All/HPVI6 & 5.5 & 5.5 & 100 & & \\
\hline
\end{tabular}


Table I (Continued)

\begin{tabular}{|c|c|c|c|c|c|c|}
\hline Author & Country & $\begin{array}{l}\text { Recruitment setting/ } \\
\text { patient characteristics }\end{array}$ & $\begin{array}{l}\text { Age, years } \\
\text { range (mean) }\end{array}$ & $\begin{array}{l}\text { Urine sampling } \\
\text { procedure }\end{array}$ & $\begin{array}{l}\text { HPV types - assays } \\
\text { (types detected) }^{a}\end{array}$ & $\begin{array}{l}\text { Total } \\
\text { sample size }\end{array}$ \\
\hline $\begin{array}{l}\text { Alameda } \\
\text { et } \mathrm{al}^{26}\end{array}$ & Spain & $\begin{array}{l}\text { Gynecological clinic patients. } \\
\text { Women with poor } \\
\text { gynecologic attention }\end{array}$ & $28-55(36)$ & $\begin{array}{l}\text { Prior to cervical } \\
\text { sampling }\end{array}$ & $\begin{array}{l}\text { PCR with MY primers } \\
\text { and papillomavirus clinical } \\
\text { array (any type }=- \text { ) }\end{array}$ & 50 \\
\hline
\end{tabular}

\begin{tabular}{|c|c|c|}
\hline $\begin{array}{l}\text { Daponte } \\
\text { et } \mathrm{al}^{28}\end{array}$ & Greece & $\begin{array}{l}\text { Colposcopy clinic referral. } \\
\text { Abnormal cytology }\end{array}$ \\
\hline \multirow{2}{*}{$\begin{array}{l}\text { Gupta } \\
\text { et } \mathrm{al}^{30}\end{array}$} & India & Invasive cancer clinic patients \\
\hline & & Healthy women (controls) \\
\hline $\begin{array}{l}\text { Song } \\
\text { et } \mathrm{al}^{35}\end{array}$ & South Korea & $\begin{array}{l}\text { Consecutively enrolled } \\
\text { gynecological clinic patients }\end{array}$ \\
\hline
\end{tabular}

26-77 (45.2)

First-void urine
prior to colposcopy
and cervical
sampling
Prior to biopsy or
cervical sampling

cervical sampling

Two weeks after cervical sampling
In house type-specific primers and commercial type-specific E6 primers for HPVI 6 and HPVI 8 In house $\mathrm{LI}$ consensus primers (any type $=-$ )

HPV DNA chip (any type $=22$ types)
77

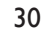

30

100

$\begin{array}{lll}\begin{array}{l}\text { Payan } \\ \text { et } \mathrm{al}^{32}\end{array} & \text { France } & \begin{array}{l}\text { Gynecological clinic patients. } \\ \text { Gynecologist referred/ } \\ \text { consulting patients }\end{array} \\ \text { Bissett } & \text { UK } & \begin{array}{l}\text { Routine colposcopy } \\ \text { clinic patients }\end{array}\end{array}$

First-void urine

after cervical

sampling

Sampled at clinic

\author{
In house LI consensus \\ primers (any type $=-$ ) \\ Modified GP primer \\ protocol \\ (HR-HPV = I 3 types)
}

$333^{\prime}$

\begin{tabular}{|c|c|c|c|c|c|}
\hline $\begin{array}{l}\text { Cuschieri } \\
\text { et } \mathrm{al}^{18}\end{array}$ & Scotland & $\begin{array}{l}\text { Recruited from a drop-in } \\
\text { sexual health service clinic }\end{array}$ & $16-25$ & Sampled at clinic & $\begin{array}{l}\text { HPV INNO-LiPA } \\
\text { (any type }=27 \text { types, } \\
\text { type-specific } 27 \text { types) }\end{array}$ \\
\hline
\end{tabular}

Notes: aRefers to the assay used to detect overall HPV prevalence; bremaining samples were excluded for other reasons; 'weighted prevalence; 'based on "Total sample size" and not "Samples with human genomic DNA;" 'HPV Genotyping Extra assay (Innogenetics); furethral samples instead of urine; 'paired urine and cervical samples from 343 females; ' $m$ median value; 'type-specific concordance calculated for HPV types for which five or more women had prevalent infection at least at one site; incomplete for various reasons, including insufficient DNA; 'kappa statistics; 'only 177 urine samples were obtained.

Abbreviations: MY, MY09/MYII/( HMB0I) primer system; GP, GP primer system; HCII, Hybrid capture II; E6, E6 primer system; Mod/severe, Moderate/Severe; Borderl, Borderline; HR, high risk; STD, sexually transmitted disease; LSIL, low-grade squamous intraepithelial lesions; HSIL, high-grade squamous intraepithelial lesions; ASCUS, atypical squamous cells of undetermined significance; CIN, cervical intraepithelial neoplasia; PCR, polymerase chain reaction; PAP, papanicolaou test; NA, not available; INNO-LIPA, (Innogenetics-line probe assay).

samples and urine samples had the lowest human genomic DNA detection rate, with $56.1 \%$ and $57.0 \%$, respectively, compared to $78.8 \%-95.1 \%$ from other urogenital sites.

\section{Technical issues affecting human genomic DNA and HPV DNA detection}

To illustrate some of the technical variability in these studies, we have listed the urine sampling procedure and the DNA detection method used (Tables 1 and 2). The most common urine sampling procedure in both female and male populations was first-void urine in contrast to mid-stream urine, which Vorsters et a ${ }^{17}$ suggested would be preferable if the goal were to analyze a maximum number of exfoliated cells. The order of urine and cervical sampling may also affect the HPV DNA detection rate in urine, and some studies have suggested that it is preferable to collect 


\begin{tabular}{|c|c|c|c|c|c|c|}
\hline $\begin{array}{l}\text { Samples with human } \\
\text { genomic DNA (\%) }\end{array}$ & $\begin{array}{l}\text { Lesions/HPV } \\
\text { types (\% lesions) }\end{array}$ & $\begin{array}{l}\text { HPV positivity } \\
\text { in urine samples (\%) }\end{array}$ & $\begin{array}{l}\text { HPV positivity in } \\
\text { cervical samples (\%) }\end{array}$ & $\begin{array}{l}\text { Concordance } \\
(\%)\end{array}$ & $\begin{array}{l}\text { Sensitivity } \\
\text { (\%) }\end{array}$ & $\begin{array}{l}\text { Specificity } \\
(\%)\end{array}$ \\
\hline \multirow[t]{5}{*}{$50(100)$} & All/any type & 22.0 & 34.0 & 80.0 & 52.9 & 93.9 \\
\hline & All/HPVI6 & 16.0 & 22.0 & 90.0 & 63.6 & 97.4 \\
\hline & LSIL/any type (32) & 6.3 & 31.3 & 75.0 & 20.0 & 100.0 \\
\hline & HSIL/any type (28) & 71.4 & 85.7 & 57.1 & 66.7 & \\
\hline & ASCUS/any type (40) & 0.0 & 0.0 & & & \\
\hline \multirow[t]{4}{*}{$77(100)$} & All/HPVI6/18 & 33.8 & 48.1 & 85.7 & 70.3 & 100.0 \\
\hline & Low grade/HPVI6/I8 (5I) & 12.8 & 28.2 & $18.7^{\mathrm{k}}$ & 45.5 & 100.0 \\
\hline & High grade/HPVI6/I8 (38) & 44.8 & 58.6 & $51.0^{k}$ & 76.5 & 100.0 \\
\hline & Cancer/HPVI6/I8 (I2) & 88.9 & 100 & $92.4^{k}$ & 88.9 & \\
\hline \multirow[t]{2}{*}{$28(93.3)$} & All/any type & 82.1 & $83.3^{d}$ & 100 & 100 & 100 \\
\hline & All/HPVI6 & 67.9 & $70.0^{\mathrm{d}}$ & 100 & 100 & 100 \\
\hline \multirow[t]{2}{*}{$30(100)$} & All/any type & 26.7 & 26.7 & 100 & 100 & 100 \\
\hline & All/HPVI6 & 16.7 & 16.7 & 100 & 100 & 100 \\
\hline \multirow[t]{12}{*}{$90(90.0)$} & All/any type & 52.2 & $70.0^{\mathrm{d}}$ & $69.3^{k}$ & & \\
\hline & All/HPVI6 & 34.4 & $38.0^{\mathrm{d}}$ & $64.0^{k}$ & 65.9 & 95.7 \\
\hline & All/HPVI8 & 3.3 & $5.0^{\mathrm{d}}$ & $58.0^{k}$ & 40.0 & 98.8 \\
\hline & CIN/any type (48) & 62.8 & $83.3^{d}$ & & & \\
\hline & CIN/HPVI6 & 37.2 & $35.4^{\mathrm{d}}$ & & & \\
\hline & CIN/HPVI8 & 4.7 & $6.3^{d}$ & & & \\
\hline & Cancer/any type (27) & 70.8 & $89.7^{d}$ & & & \\
\hline & Cancer/HPVI6 & 50.0 & $62.1^{\mathrm{d}}$ & & & \\
\hline & Cancer/HPVI8 & 4.2 & $6.9^{\mathrm{d}}$ & & & \\
\hline & C.Cervicitis/any type (26) & 13.0 & $17.4^{\mathrm{d}}$ & & & \\
\hline & C.Cervicitis/HPVI6 & 8.7 & $13.0^{\mathrm{d}}$ & & & \\
\hline & C.Cervicitis/HPVI8 & 0 & 0 & & & \\
\hline $177(100)$ & All/any type & 37.3 & $45.0^{\mathrm{d}}$ & $93.2^{\mathrm{k}}$ & 91.2 & 96.3 \\
\hline \multirow[t]{10}{*}{$253(95.8)$} & All/HR-HPV & 70.4 & 80.6 & $57.9^{k}$ & 83.8 & 85.7 \\
\hline & All/HPVI6/I8 & 30.0 & 38.3 & $76.5^{\mathrm{k}}$ & 75.3 & 98.1 \\
\hline & Normal/HR-HPV (20) & 55.3 & 57.4 & & 85.1 & 85.2 \\
\hline & Normal/HPVI6/I8 & 19.1 & 19.1 & & 88.9 & 97.4 \\
\hline & Borderl/mild/HR-HPV (50) & 70.8 & 82.5 & & 83.8 & 90.5 \\
\hline & Borderl/mild/HPVI6/I8 & 26.7 & 35.0 & & 76.2 & 100 \\
\hline & Mod/severe/HR-HPV (30) & 79.2 & 91.7 & & 84.8 & 83.3 \\
\hline & Mod/severe/HPVI6/I8 & 43.1 & 56.9 & & 70.7 & 93.5 \\
\hline & All/any type & 66.7 & 58.9 & $59.8^{k}$ & 90.6 & 67.6 \\
\hline & Type-specific HPV & & & 70.8 & & \\
\hline
\end{tabular}

the urine sample first (reviewed in Sehgal et al). ${ }^{21}$ In the female populations listed in Table 1, urine sampling was not always carried out prior to cervical sampling and was sometimes done after washing the genitals. For HPV DNA detection, variants of the MY09/MY11 primer were most commonly used, but other primers, such as general primer $\mathrm{GP}^{+} / 6^{+}$, in-house primers, E6-primers, hybrid capture II, and DNA chip assays were also used. In summary, the technical variability may contribute substantially to HPV DNA detection rates.

\section{Discussion}

According to the World Health Organization (WHO), 33 countries have implemented HPV vaccination as part of their national immunization program. ${ }^{41}$ Generally, vaccination is provided to girls, and it is therefore recommended to 


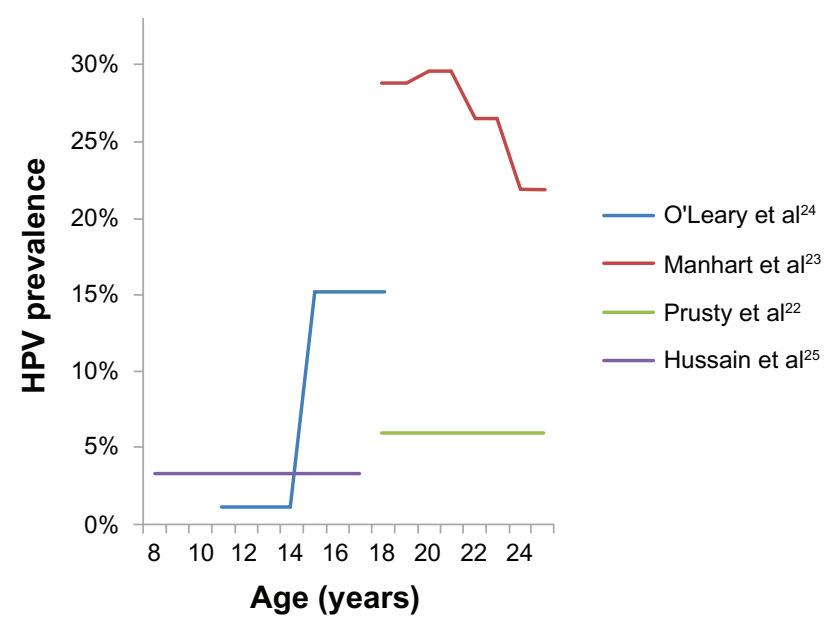

Figure I Human papillomavirus prevalence by age in urine samples from asymptomatic populations.

Abbreviation: HPV, human papillomavirus.

monitor the impact of vaccination on HPV prevalence among female adolescents. ${ }^{15}$ In August 2012, we identified four publications that reported HPV prevalence in urine samples from asymptomatic female adolescents. HPV prevalence has been strongly associated with age, being nearly nonexistent in preadolescents, gradually increasing with sexual activity among adolescents, and generally peaking around 25 years of age ${ }^{42}$ However, regional differences in HPV prevalence do exist. ${ }^{43}$ Therefore, the first step when monitoring HPV prevalence is to establish the baseline HPV prevalence in the relevant age groups. To date, only Hussain et al and O'Leary et al reported baseline HPV prevalence based on urine samples from a large unvaccinated adolescent population. ${ }^{24,25}$ The Hussain et $a 1^{25}$ study from India used self-collected urine

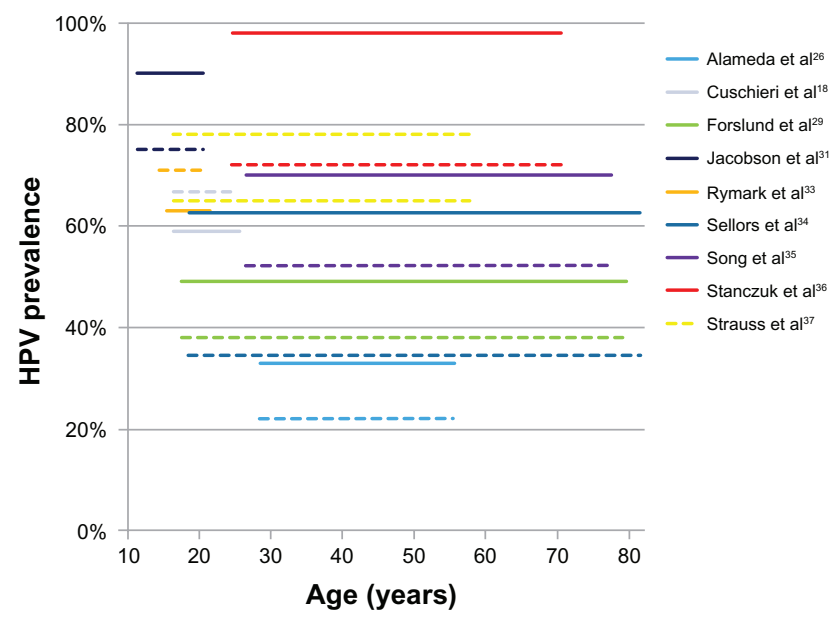

Figure 2 Human papillomavirus prevalence by age from paired urine and cervical samples from symptomatic populations (any HPV).

Notes: Dashed line represents urine samples, solid line represents cervical samples. Abbreviation: HPV, human papillomavirus. samples from healthy children attending public school and achieved a $57.3 \%$ participation rate. O'Leary et $\mathrm{al}^{24}$ analyzed urine samples from 11-18-year-old school and college males (1121) and females (1341) in Scotland 4 months before vaccination was introduced in the national immunization schedule in 2008. A limitation of the study was that the estimated response rate for providing a urine sample was as low as $14 \%$. While the low response rate can introduce bias and lead to erroneous estimates of the overall HPV prevalence, it was not directly related to the sampling method and probably reflects a general challenge to achieve high response rates in this age group. This, in turn, could partially explain why only few studies measuring HPV prevalence in urine samples from female adolescents in the general population have been performed and published.

The five studies that reported HPV prevalence by lesion severity showed a similar association for paired urine and cervical samples, with higher HPV prevalence in the most severe lesions. There were major variations in HPV prevalence in urine samples across the studies. This is to some degree related to regional differences in HPV prevalence, ${ }^{43}$ the age distribution of the different study populations, ${ }^{43}$ and the setting in which the women were recruited. There were also differences in sampling procedures and HPV detection methods, including the number of types detected by a given assay. It is therefore not possible, as Vorsters et a ${ }^{17}$ pointed out, to perform a meta-analysis on the present urine-based HPV prevalence studies.

Detection of human genomic DNA is commonly used to control for the adequacy of samples for HPV detection. Studies including female populations showed a high detection rate of human genomic DNA in urine samples ( $83 \%-100 \%)$, while male populations showed a larger range of detection rates (30\%-100\%). In an HPV monitoring setting, a low human genomic DNA detection rate would lead to reduced coverage and create a concern of bias in HPV estimates. In general, studies on female populations in this review indicated that high detection rates of human genomic DNA are feasible.

In cervical screening the main focus is to detect HPV or cervical abnormalities at the individual level, while population-based HPV prevalence is used more in a monitoring or epidemiological setting. Therefore, although prevalence in urine samples was lower than in cervical samples in the studies included in this review, monitoring by regular urine measurements over time may still be a useful way of identifying shifts in HPV prevalence due to imparted immunity against vaccine HPV types. However, the differences in HPV concordance of paired urine and cervical samples illustrates 


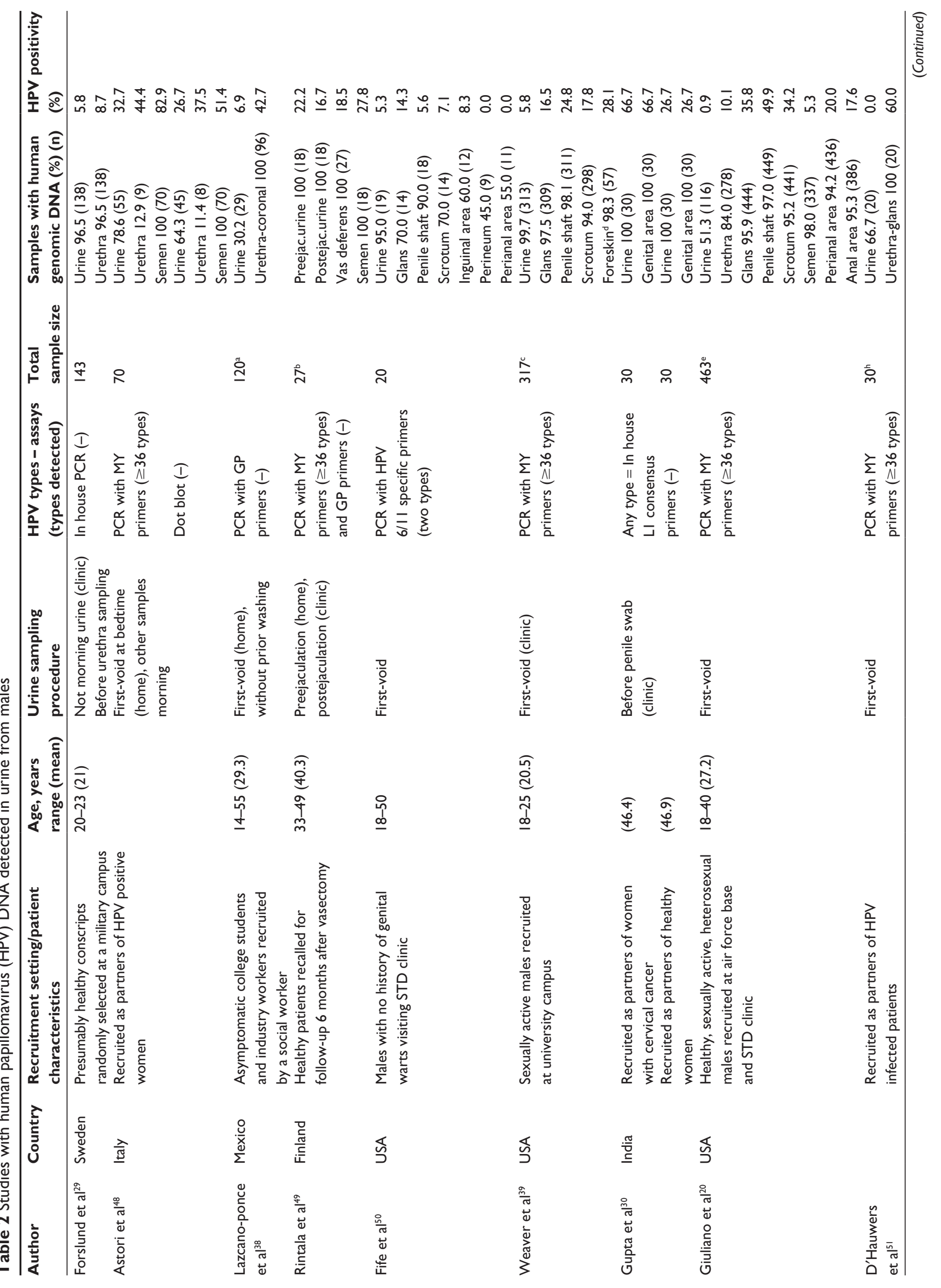




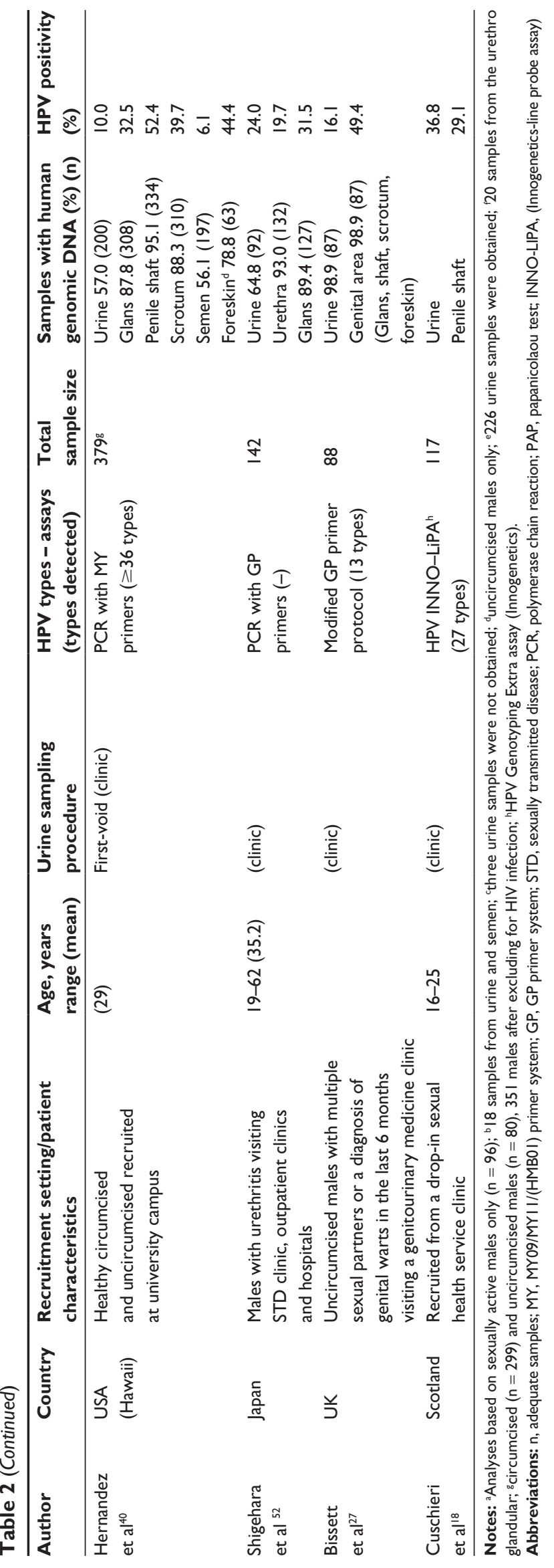

that HPV positivity in urine should be interpreted independently of the cervix and that care should be taken when inferring that a similar change is taking place in the cervix. On the other hand, HPV detection in urine samples could be considered an independent measurement of the impact of HPV vaccination but it would have only limited public health interest. Furthermore, we observed that HPV negativity in the cervix commonly predicted an HPV-negative result in the urine as well, while HPV positivity in the cervix less commonly predicted HPV positivity in the urine. Although Daponte et al showed an increased concordance with increased lesion severity for any HPV type, other studies like Alameda et al, ${ }^{26}$ Rymark et al, ${ }^{33}$ and Gupta et $a l^{30}$ showed a relatively high concordance, even in populations where HPV prevalence is low. The variability of HPV16/18-specific concordance, the types included in both of the available HPV vaccines, further exemplifies the uncertainty of using urine samples to estimate future changes in the incidence of cervical lesions.

The most comprehensive monitoring of changes in HPV prevalence would be carried out by establishing baseline HPV prevalence before measuring any impact of vaccination as well as regular measurements of HPV prevalence in both vaccinated and unvaccinated females and males. The age group (or groups) and sample size to include in HPV monitoring should be carefully selected to assure there is enough statistical power to identify changes in overall HPV prevalence as well as HPV type-specific changes. Models suggest that there will be a significant reduction in the prevalence of vaccine HPV types in males in the future because after vaccination, fewer girls will transmit HPV to their male partners. ${ }^{44}$ Monitoring HPV prevalence in males could therefore be a near-term end point that could also help to estimate the effect of herd immunity. However, monitoring HPV prevalence in males presents several challenges. The differences in HPV prevalence across different urogenital sites illustrates that no single site repeatedly shows the highest HPV DNA detection rate and that urine in particular has a relatively low HPV DNA detection rate compared to other sites. In addition, male urine generally has a lower detection rate for human genomic DNA than samples from other urogenital sites. With lower detection rates for human genomic DNA, a larger sample size would be needed to detect changes with the same power as other urogenital sites. Based on these aspects, other anatomical sites seem more favorable for males.

Monitoring changes in HPV prevalence requires regular prevalence measurements over many years. A protocol with sufficient detail on technical and practical issues that influence HPV detection is therefore necessary to ensure 
comparability between these measurements. This includes, among other issues, urine sampling procedure, handling of samples, extraction of DNA, and assay used for HPV genotyping. ${ }^{17}$ In addition it might be useful to store an extra aliquot of extracted DNA from each regular measurement to be able to perform HPV genotyping on all DNA collected from urine samples over many years. This would also allow for using any novel genotyping technology that may have developed during the monitoring period. Information on more aspects of HPV monitoring can also be found in the Human Papillomavirus Laboratory Manual issued in 2009 by the WHO HPV Laboratory Network (WHO HPV LabNet). ${ }^{45}$ This manual covers guidance on specimen collection and handling for HPV testing, with the aim to assist in establishing the laboratory support required for implementation and monitoring of HPV vaccination programs. Several of the WHO HPV LabNet members are actively undertaking studies of HPV detection in urine, and a leading role for the WHO HPV LabNet in further standardizing and optimizing the technology for HPV detection in urine seems appropriate. ${ }^{18,45,46}$

This is the first review that focuses solely on the use of urine to monitor changes in HPV prevalence in an asymptomatic population. The major shortcoming of this review is that, to date, there are few studies on the topic. We have therefore included studies from symptomatic populations and older populations that used urine for purposes other than monitoring, although these are not comparable to asymptomatic adolescents in all aspects. In addition, the studies highlighted in the present review as well as in the reviews of Vorsters et $\mathrm{al}^{17}$ and Seghal et al, ${ }^{21}$ showed that the large variability in sampling and genotyping methodology make direct comparisons of data, like concordance, inaccurate. ${ }^{17,21}$

Assuming a future reduction in overall HPV prevalence and vaccine HPV type-specific prevalence and using urine testing as a monitoring method, care should be taken when interpreting the data. Indeed the data may not necessarily mimic the true HPV distribution in the cervix nor estimate the expected reductions in cervical cancer and high-grade lesions, as indicated by variable vaccine HPV type-specific (HPV16/18) concordance between paired urine and cervical samples. There is great scientific and political interest in monitoring the early effects of HPV vaccination in the general population. However, monitoring HPV prevalence as an early measurement of vaccine impact is only possible in a few countries as substantial financial and human resources are needed as well as a 5-10-year commitment in order to demonstrate results. ${ }^{16}$

\section{Conclusion}

Urine is an adequate alternative biospecimen for monitoring HPV prevalence in female adolescents to determine the early effect of HPV vaccination on a population level. Strategies for recruitment should be optimized to avoid low response rates, sampling and HPV detection protocols should be detailed and standardized to ensure comparability, and importantly, care should be taken when extrapolating findings to the cervix. In males, urine samples do not seem to be optimal for monitoring HPV prevalence due to a low human genomic DNA content compared to other urogenital sites. Although urine sampling has some advantages and is the only relevant option for sampling the general population in the youngest age groups, it also has several disadvantages, most importantly the fact that HPV prevalence in urine is only a distant measure of the main end point of vaccine impact, cervical cancer. In each situation the costs and benefits of HPV DNA detection in urine, compared to alternative monitoring options, should be carefully considered. ${ }^{16,47}$

\section{Acknowledgments}

We thank Elisabete Weiderpass for initiating the study and critically revising the manuscript and Trudy Perdrix-Thoma for editorial assistance.

\section{Disclosure}

$\mathrm{EE}$ and $\mathrm{CO}$ report no conflicts of interest in this work. MN has received research funding from Merck through the institution.

\section{References}

1. Walboomers JM, Jacobs MV, et al. Human papillomavirus is a necessary cause of invasive cervical cancer worldwide. J Pathol. 1999;189(1): $12-19$.

2. zur Hausen H. Papillomavirus infections - a major cause of human cancers. Biochim Biophys Acta. 1996;1288(2):F55-F78.

3. Munoz N, Bosch FX, de Sanjose S, et al. Epidemiologic classification of human papillomavirus types associated with cervical cancer. $N$ Engl J Med. 2003;348(6):518-527.

4. Bosch FX, de Sanjose S. Chapter 1: Human papillomavirus and cervical cancer - burden and assessment of causality. J Natl Cancer Inst Monogr. 2003;31:3-13.

5. Ault KA. Effect of prophylactic human papillomavirus L1 virus-likeparticle vaccine on risk of cervical intraepithelial neoplasia grade 2, grade 3 , and adenocarcinoma in situ: a combined analysis of four randomised clinical trials. Lancet. 2007;369(9576):1861-1868.

6. Paavonen J, Jenkins D, Bosch FX, et al. Efficacy of a prophylactic adjuvanted bivalent $\mathrm{L} 1$ virus-like-particle vaccine against infection with human papillomavirus types 16 and 18 in young women: an interim analysis of a phase III double-blind, randomised controlled trial. Lancet. 2007;369(9580):2161-2170.

7. Food and Drug Administration. Center for Biologics and Evaluation and Research, Office of Vaccines and Related Product Applications. Available from: http://www.fda.gov/BiologicsBloodVaccines/Vaccines/ ApprovedProducts/ucm094042.htm. Accessed September 17, 2012. 
8. Villa LL. HPV prophylactic vaccination: The first years and what to expect from now. Cancer Lett. 2011;305(2):106-112.

9. Paavonen J, Naud P, Salmeron J, et al. Efficacy of human papillomavirus (HPV)-16/18 AS04-adjuvanted vaccine against cervical infection and precancer caused by oncogenic HPV types (PATRICIA): final analysis of a double-blind, randomised study in young women. Lancet. 2009;374(9686):301-314.

10. Block SL, Brown DR, Chatterjee A, et al. Clinical trial and postlicensure safety profile of a prophylactic human papillomavirus (types $6,11,16$, and 18) 11 virus-like particle vaccine. Pediatr Infect Dis J. 2010;29(2):95-101.

11. Descamps D, Hardt K, Spiessens B, et al. Safety of human papillomavirus (HPV)-16/18 AS04-adjuvanted vaccine for cervical cancer prevention: a pooled analysis of 11 clinical trials. Hum Vaccin. 2009;5(5): 332-340.

12. Giuliano AR, Lazcano-Ponce E, Villa L, et al. Impact of baseline covariates on the immunogenicity of a quadrivalent (types $6,11,16$, and 18) human papillomavirus virus-like-particle vaccine. J Infect Dis. 2007;196(8):1153-1162.

13. Munoz N, Manalastas R Jr, Pitisuttithum P, et al. Safety, immunogenicity, and efficacy of quadrivalent human papillomavirus (types $6,11,16,18$ ) recombinant vaccine in women aged $24-45$ years: a randomised, double-blind trial. Lancet. 2009;373(9679):1949-1957.

14. Romanowski B, de Borba PC, Naud PS, et al. Sustained efficacy and immunogenicity of the human papillomavirus (HPV)-16/18 AS04adjuvanted vaccine: analysis of a randomised placebo-controlled trial up to 6.4 years. Lancet. 2009;374(9706):1975-1985.

15. Ferguson M, Wilkinson DE, Zhou T. WHO meeting on the standardization of HPV assays and the role of the WHO HPV Laboratory Network in supporting vaccine introduction held on January 24-25, 2008, Geneva, Switzerland. Vaccine. 2009;27(3):337-347.

16. Monitoring the coverage and impact of human papillomavirus vaccine - report of WHO meeting, Nov 2009. Wkly Epidemiol Rec. 2010;85(25):237-243.

17. Vorsters A, Micalessi I, Bilcke J, Ieven M, Bogers J, Van Damme P. Detection of human papillomavirus DNA in urine. A review of the literature. Eur J Clin Microbiol Infect Dis. 2012;31(5):627-640.

18. Cuschieri K, Nandwani R, McGough P, et al. Urine testing as a surveillance tool to monitor the impact of HPV immunization programs. J Med Virol. 2011;83(11):1983-1987.

19. Dunne EF, Nielson CM, Stone KM, Markowitz LE, Giuliano AR. Prevalence of HPV infection among men: A systematic review of the literature. J Infect Dis. 2006;194(8):1044-1057.

20. Giuliano AR, Nielson CM, Flores R, et al. The optimal anatomic sites for sampling heterosexual men for human papillomavirus (HPV) detection: the HPV detection in men study. J Infect Dis. 2007;196(8): 1146-1152.

21. Sehgal A, Gupta S, Parashari A, Sodhani P, Singh V. Urine HPV-DNA detection for cervical cancer screening: prospects and prejudices. J Obstet Gynaecol. 2009;29(7):583-589.

22. Prusty BK, Kumar A, Arora R, Batra S, Das BC. Human papillomavirus (HPV) DNA detection in self-collected urine. Int J Gynaecol Obstet. 2005;90(3):223-227.

23. Manhart LE, Holmes KK, Koutsky LA, et al. Human papillomavirus infection among sexually active young women in the United States: Implications for developing a vaccination strategy. Sex Transm Dis. 2006;33(8):502-508.

24. O'Leary MC, Sinka K, Robertson C, et al. HPV type-specific prevalence using a urine assay in unvaccinated male and female 11- to 18-year olds in Scotland. Br J Cancer. 2011;104(7):1221-1226.

25. Hussain S, Bharadwaj M, Nasare V, et al. Human papillomavirus infection among young adolescents in India: impact of vaccination. $J$ Med Virol. 2012;84(2):298-305.

26. Alameda F, Bellosillo B, Fuste P, et al. Human papillomavirus detection in urine samples: an alternative screening method. $J$ Low Genit Tract Dis. 2007;11(1):5-7.

27. Bissett SL, Howell-Jones R, Swift C, et al. Human papillomavirus genotype detection and viral load in paired genital and urine samples from both females and males. J Med Virol. 2011;83(10):1744-1751.
28. Daponte A, Pournaras S, Mademtzis I, et al. Evaluation of high-risk human papillomavirus types PCR detection in paired urine and cervical samples of women with abnormal cytology. J Clin Virol. 2006;36(3): 189-193.

29. Forslund O, Hansson BG, Rymark P, Bjerre B. Human papillomavirus DNA in urine samples compared with that in simultaneously collected urethra and cervix samples. J Clin Microbiol. 1993;31(8):1975-1979.

30. Gupta A, Arora R, Gupta S, et al. Human papillomavirus DNA in urine samples of women with or without cervical cancer and their male partners compared with simultaneously collected cervical/penile smear or biopsy specimens. J Clin Virol. 2006;37(3):190-194.

31. Jacobson DL, Womack SD, Peralta L, et al. Concordance of human papillomavirus in the cervix and urine among inner city adolescents. Pediatr Infect Dis J. 2000;19(8):722-728.

32. Payan C, Ducancelle A, Aboubaker MH, et al. Human papillomavirus quantification in urine and cervical samples by using the Mx4000 and LightCycler general real-time PCR systems. J Clin Microbiol. 2007;45(3):897-901.

33. Rymark P, Forslund O, Hansson BG, Lindholm K. Genital HPV infection not a local but a regional infection: experience from a female teenage group. Genitourin Med. 1993;69(1):18-22.

34. Sellors JW, Lorincz AT, Mahony JB, et al. Comparison of self-collected vaginal, vulvar and urine samples with physician-collected cervical samples for human papillomavirus testing to detect high-grade squamous intraepithelial lesions. CMAJ. 2000;163(5):513-518.

35. Song ES, Lee HJ, Hwang TS. Clinical efficacy of human papillomavirus DNA detection in urine from patients with various cervical lesions. J Korean Med Sci. 2007;22(1):99-104.

36. Stanczuk GA, Kay P, Allan B, et al. Detection of human papillomavirus in urine and cervical swabs from patients with invasive cervical cancer. J Med Virol. 2003;71(1):110-114.

37. Strauss S, Jordens JZ, McBride D, et al. Detection and typing of human papillomavirus DNA in paired urine and cervical scrapes. Eur $J$ Epidemiol. 1999;15(6):537-543.

38. Lazcano-Ponce E, Herrero R, Munoz N, et al. High prevalence of human papillomavirus infection in Mexican males: comparative study of penile-urethral swabs and urine samples. Sex Transm Dis. 2001;28(5): 277-280.

39. Weaver BA, Feng Q, Holmes KK, et al. Evaluation of genital sites and sampling techniques for detection of human papillomavirus DNA in men. J Infect Dis. 2004;189(4):677-685.

40. Hernandez BY, Wilkens LR, Zhu X, et al. Circumcision and human papillomavirus infection in men: a site-specific comparison. $J$ Infect Dis. 2008;197(6):787-794.

41. Arbyn M, Sanjose SD, Saraiya M, et al. EUROGIN 2011 roadmap on prevention and treatment of HPV-related disease. Int $J$ Cancer. 2012;131(9):1969-1982.

42. Bruni L, Diaz M, Castellsague X, Ferrer E, Bosch FX, de Sanjose S. Cervical human papillomavirus prevalence in 5 continents: meta-analysis of 1 million women with normal cytological findings. $J$ Infect Dis. 2010;202(12):1789-1799.

43. de Sanjose S, Diaz M, Castellsague X, et al. Worldwide prevalence and genotype distribution of cervical human papillomavirus DNA in women with normal cytology: a meta-analysis. Lancet Infect Dis. 2007;7(7): 453-459.

44. Chesson HW, Ekwueme DU, Saraiya M, Dunne EF, Markowitz LE. The cost-effectiveness of male HPV vaccination in the United States. Vaccine. 2011;29(46):8443-8450.

45. Organization WH. WHO HPV Laboratory Network. Available from: http://www.who.int/biologicals/vaccines/hpv/en/index.html. Accessed November 23, 2012.

46. Johnson AM, Mercer $\mathrm{CH}$, Beddows $\mathrm{S}$, et al. Epidemiology of, and behavioural risk factors for, sexually transmitted human papillomavirus infection in men and women in Britain. Sex Transm Infect. 2012;88(3): 212-217.

47. Wong CA, Saraiya M, Hariri S, et al. Approaches to monitoring biological outcomes for HPV vaccination: challenges of early adopter countries. Vaccine. 2011;29(5):878-885. 
48. Astori G, Pipan C, Muffato G, Botta GA. Detection of HPV-DNA in semen, urine and urethral samples by dot blot and PCR. New Microbiol. 1995;18(2):143-149.

49. Rintala MA, Pollanen PP, Nikkanen VP, Grenman SE, Syrjanen SM. Human papillomavirus DNA is found in the vas deferens. J Infect Dis. 2002;185(11):1664-1667.

50. Fife KH, Coplan PM, Jansen KU, et al. Poor sensitivity of polymerase chain reaction assays of genital skin swabs and urine to detect HPV 6 and 11 DNA in men. Sex Transm Dis. 2003;30(3):246-248.
51. D’Hauwers K, Depuydt C, Bogers JP, et al. Urine versus brushed samples in human papillomavirus screening: study in both genders. Asian J Androl. 2007;9(5):705-710.

52. Shigehara K, Sasagawa T, Kawaguchi S, et al. Prevalence of human papillomavirus infection in the urinary tract of men with urethritis. Int J Urol. 2010;17(6):563-568.
Clinical Epidemiology

\section{Publish your work in this journal}

Clinical Epidemiology is an international, peer-reviewed, open access journal focusing on disease and drug epidemiology, identification of risk factors and screening procedures to develop optimal preventative initiatives and programs. Specific topics include: diagnosis, prognosis, treatment, screening, prevention, risk factor modification, systematic

Submit your manuscript here: http://www.dovepress.com/clinical-epidemiology-journal

\section{Dovepress}

reviews, risk \& safety of medical interventions, epidemiology \& biostatical methods, evaluation of guidelines, translational medicine, health policies \& economic evaluations. The manuscript management system is completely online and includes a very quick and fair peer-review system, which is all easy to use. 